\title{
Magnetohydrodynamic Stability of Streaming Jet Pervaded Internally by Varying Transverse Magnetic Field
}

\begin{abstract}
Alfaisal A. Hasan
Basic and Applied Sciences Department, College of Engineering and Technology, Arab Academy for Science E Technology and Maritime Transport (AASTMT), P.O. Box 2033 Elhorria, Cairo, Egypt

Correspondence should be addressed to Alfaisal A. Hasan, alfaisal772001@yahoo.com

Received 9 October 2011; Accepted 12 December 2011

Academic Editor: Alexei Mailybaev

Copyright (C) 2012 Alfaisal A. Hasan. This is an open access article distributed under the Creative Commons Attribution License, which permits unrestricted use, distribution, and reproduction in any medium, provided the original work is properly cited.

The Magnetohydrodynamic stability of a streaming cylindrical model penetrated by varying transverse magnetic field has been discussed. The problem is formulated, the basic equations are solved, upon appropriate boundary conditions the eigenvalue relation is derived and discussed analytically, and the results are verified numerically. The capillary force is destabilizing in a small axisymmetric domain $0<x<1$ and stabilizing otherwise. The streaming has a strong destabilizing effect in all kinds of perturbation. The toroidal varying magnetic field interior the fluid has no direct effect at all on the stability of the fluid column. The axial exterior field has strong stabilizing effect on the model. The effect of all acting forces altogether could be identified via the numerical analysis of the stability theory of the present model.
\end{abstract}

\section{Introduction}

The classical stability analysis of a full fluid jet has been extensively studied (cf. Rayleigh [1], Chandrasekhar [2], Robert [3], Chenng [4], Kendall [5], and Drazin and Reid) [6] and documented by Radwan [7]. The latter author studied the hydromagnetic stability of a fluid jet pervaded by uniform magnetic field for axisymmetric perturbation. Radwan et al. [8] developed the magnetohydrodynamic stability of that model for all axisymmetric and nonaxisymmetric modes subject to electromagnetic forces generated due to constant magnetic field. The stability of different models under the action of self-gravitating force in addition to other forces has been elaborated by Radwan and Hasan [9, 10]. Hasan [11] has discussed the stability of oscillating streaming fluid cylinder subject to combined effect of the capillary, self-gravitating, and electrodynamic forces for all axisymmetric and 
nonaxisymmetric perturbation modes. The purpose of the present work is to investigate the magnetodynamic stability of a fluid jet pervaded by transverse varying magnetic field while its surrounding tenuous medium is penetrated by uniform magnetic field.

\section{Basic State}

We consider an incompressible, inviscid, and conducting fluid column of radius $R_{o}$ in the basic state.

A transverse varying magnetic field is assumed to be pervaded interior the fluid, namely,

$$
\underline{H}_{o}=\left(0, \frac{H_{o} r}{R_{o}}, 0\right)
$$

The surrounding tenuous medium of the fluid jet is penetrated by the axial homogenous magnetic field:

$$
\underline{H}_{o}^{t n}=\left(0,0, \alpha H_{o}\right)
$$

where $\alpha$ is parameter while $H_{o}$ is the intensity of the transverse magnetic field across the fluid surface at $r=R_{o}$. The fluid streams in the initial state with velocity:

$$
\underline{u}=\left(0,0, U_{o}\right),
$$

where $U_{o}$ is (uniform) the amplitude of the velocity $\underline{u}$. The components of $\underline{H}_{o}$ and $\underline{H}_{o}^{t n}$ are considered along the utilizing cylindrical coordinates $(r, \varphi, z)$ with the $Z$-axis coinciding with the axis of the cylinder (see Figure 1).

The basic magnetodynamic equations appropriate for studying such a problem may be formulated as follows interior the fluid cylinder:

$$
\begin{gathered}
\rho \frac{d \underline{u}}{d t}=-\nabla P+\mu(\nabla \wedge \underline{H}) \wedge \underline{H}, \\
\nabla \cdot \underline{u}=0, \\
\frac{\partial \underline{H}}{\partial t}=\nabla \wedge(\underline{u} \wedge \underline{H}), \\
\nabla \cdot \underline{H}=0, \\
P_{S}=T\left(\frac{1}{r_{1}}+\frac{1}{r_{2}}\right)=T(\nabla \cdot \underline{\hat{N}}),
\end{gathered}
$$




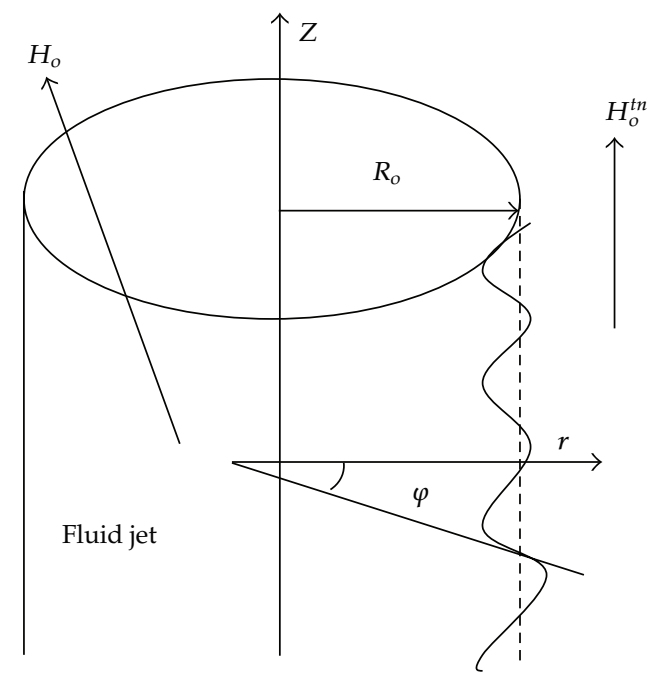

Figure 1: Sketch for MHD fluid jet.

where $r_{1}$ and $r_{2}$ are the principle radii of curvature and $T$ is the surface tension coefficient. $\underline{N}_{S}$ is, the unit outward vector normal to the surface, given by

$$
\underline{\hat{N}}_{s}=\frac{\nabla f(r, \varphi, z ; t)}{|\nabla f(r, \varphi, z ; t)|}
$$

The space $\nabla$ and the time $d / d t$ operators are given by

$$
\nabla \equiv\left(\frac{\partial}{\partial r}, \frac{1}{r} \frac{\partial}{\partial \varphi}, \frac{\partial}{\partial z}\right), \quad \frac{d}{d t}=\frac{\partial}{\partial t}+(\underline{u} \cdot \nabla)
$$

Here $\rho, \underline{u}$, and $P$ are the fluid mass density, velocity vector and kinetic pressure, $\underline{H}$ and $\underline{H}^{\text {tn }}$ are the magnetic field intensity of fluid and of tenuous medium surrounding the fluid jet. $\mu$ is the magnetic field permeability coefficient.

In the surrounding tenuous medium outside the fluid jet, the basic equations are

$$
\nabla \cdot \underline{H}^{t n}=0,
$$

$$
\nabla \wedge \underline{H}^{t n}=0, \quad \text { there is no current. }
$$

\section{Unperturbed State}

The unperturbed state is studied and the kinetic fluid pressure $P_{o}$ is identified.

From now on, the quantities with index o refer to equilibrium quantities. 
In such state, (2.4) gives

$$
\nabla\left(P_{o}+\frac{\mu}{2} \frac{H_{o}^{2} r^{2}}{R_{o}^{2}}\right)=0
$$

From which, we get

$$
P_{o}+\frac{\mu}{2} \frac{H_{o}^{2} r^{2}}{R_{o}^{2}}=k \text { (const.). }
$$

By applying the balance of the pressure across the fluid tenuous interface at $r=R_{o}$, we finally obtain

$$
P_{o}=\frac{T}{R_{o}}+\frac{\mu H_{o}^{2}}{2}\left(\alpha^{2}-1\right)
$$

where $\alpha$ satisfies the restriction $\alpha \geq 1$ in order that the pressure $P_{o}$ must be positive as we neglect the surface tension contribution in the unperturbed state.

\section{Perturbation Analysis}

For small departures from the initial state, every physical quantity could be expressed as

$$
Q(r, \varphi, z ; t)=Q_{o}(r)+\varepsilon(t) Q_{1}(r, \varphi, z)
$$

where $Q$ stands for $\underline{u}, P, \underline{H}$, and $\underline{H}^{t n}$ while, $\varepsilon(t)$ is the amplitude of the perturbation, (cf. [2]) being

$$
\varepsilon(t)=\varepsilon_{o} \exp (\sigma t)
$$

Here $\sigma$ is the growth rate at time $t$ and $\varepsilon_{o}(=\varepsilon$ at $t=0)$ that is the initial amplitude. 
By inserting the expansion (4.1) into (2.4)-(2.11), the linearized perturbation equations are given by

$$
\begin{gathered}
\frac{\partial \underline{u}_{1}}{\partial t}+\left(\underline{u}_{o} \cdot \nabla\right) \underline{u}_{1}-\frac{\mu}{\rho}\left(\left(\underline{H}_{1} \cdot \nabla\right) \underline{H}_{o}+\left(\underline{H}_{o} \cdot \nabla\right) \underline{H}_{1}\right)=-\nabla \prod \prod_{1^{\prime}} \\
\nabla \cdot \underline{u}_{1}=0, \\
\frac{\partial \underline{H}_{1}}{\partial t}=\left(\underline{H}_{o} \cdot \nabla\right) \underline{u}_{1}-\left(\underline{u}_{1} \cdot \nabla\right) \underline{H}_{o}-\left(\underline{u}_{o} \cdot \nabla\right) \underline{H}_{1}, \\
\nabla \cdot \underline{H}_{1}=0, \\
\nabla \cdot \underline{H}_{1}^{t n}=0, \\
\nabla \wedge \underline{H}_{1}^{t n}=0, \\
P_{1 s}=\frac{-T}{R_{o}^{2}}\left(R_{1}+\frac{\partial^{2} R_{1}}{\partial \varphi^{2}}+R_{o}^{2} \frac{\partial^{2} R_{1}}{\partial z^{2}}\right),
\end{gathered}
$$

where $\rho \prod_{1}\left(=P_{o}+(\mu / 2)(\underline{H} \cdot \underline{H})_{1}\right)$ (see [3]) is the total magnetohydrodynamics pressure which is the sum of kinetic and magnetic pressures.

Based on the expansion (4.1), the perturbed fluid-tenuous interface may be expressed as

$$
r=R_{o}+R_{1}(\varphi, z, t)
$$

with

$$
R_{1}=\varepsilon_{o} \exp (i(k z+m \varphi)+\sigma t)
$$

Here $R_{1}$ is the elevation of the surface wave measured from the unperturbed position, where $k$ is the longitudinal wavenumber and $m$ is the azimuthal wavenumber. By an appeal to the perturbation technique, we may write

$$
Q_{1}(r, \varphi, z)=Q_{1}^{*}(r) \exp (i(k z+m \varphi))
$$

Consequently, the linearized system of (4.3)-(4.8) is solved and the fluctuation parts are determined.

In the radial direction, we obtain

$$
\begin{gathered}
\left(\sigma+i k U_{o}\right) u_{1 r}=\frac{\mu H_{o}}{\rho R_{o}}\left(i m H_{1 r}\right)-\frac{\partial \prod_{1}}{\partial r} \\
\left(\sigma+i k U_{o}\right) H_{1 r}=\frac{i m H_{o}}{R_{o}}\left(u_{1 r}\right) .
\end{gathered}
$$


In the transverse direction, we have

$$
\begin{gathered}
\left(\sigma+i k U_{o}\right) u_{1 \varphi}=\frac{-i m \prod_{1}}{r}+\frac{\mu}{\rho R_{o}}\left(i m H_{1 \varphi}+H_{1 r}\right) H_{o} \\
\left(\sigma+i k U_{o}\right) H_{1 \varphi}=\frac{i m H_{o}}{R_{o}} u_{1 \varphi}-\frac{H_{o}}{R_{o}} u_{1 r} .
\end{gathered}
$$

In the longitudinal $z$-direction, we get

$$
\begin{gathered}
\left(\sigma+i k U_{o}\right) u_{1 z}=-i k \prod_{1}+\frac{i \mu H_{o} m}{\rho R_{o}} H_{1 z} \\
\left(\sigma+i k U_{o}\right) H_{1 z}=\frac{i m H_{o}}{R_{o}} u_{1 z}
\end{gathered}
$$

From (4.13)-(4.15), we obtain

$$
\begin{aligned}
& u_{1 r}=\frac{-\left(\sigma+i k U_{o}\right)}{\left(\left(\sigma+i k U_{o}\right)^{2}+\mu H_{o}^{2} m^{2} / \rho R_{o}^{2}\right)} \frac{\partial \prod_{1}}{\partial r}, \\
& u_{1 \varphi}=\frac{-i m\left(\sigma+i k U_{o}\right)}{\left(\left(\sigma+i k U_{o}\right)^{2}+\mu H_{o}^{2} m^{2} / \rho R_{o}^{2}\right)} \frac{\prod_{1}}{r}, \\
& u_{1 r}=\frac{-i k\left(\sigma+i k U_{o}\right)}{\left(\left(\sigma+i k U_{o}\right)^{2}+\mu H_{o}^{2} m^{2} / \rho R_{o}^{2}\right)} \prod_{1} .
\end{aligned}
$$

By taking the divergence of (4.3) and using (4.4), we obtain

$$
\nabla^{2} \prod_{1}=0
$$

Apart from the singular solution, the finite solution (as $r \rightarrow 0$ ) of this equation is given by

$$
\prod_{1}=A I_{m}(k r) \exp (\sigma t+i(k z+m \varphi)) \text {. }
$$

Now (4.8) means that $\underline{H}_{1}^{\text {tn }}$ could be derived from a scalar function, say $\phi_{1}$, such that

$$
H_{1}^{t n}=-\nabla \phi_{1}
$$

Combining (4.19) with (4.7), we get

$$
\nabla^{2} \phi_{1}=0
$$

The nonsingular solution of (4.20) is identified and given by

$$
H_{1}^{t n}=\nabla\left[B K_{m}(k r) \exp (\sigma t+i(k z+m \varphi))\right] .
$$


Here $I_{m}(k r)$ and $K_{m}(k r)$ are the modified Bessel functions, respectively, of the first and second kind of order $m$ while $A$ and $B$ are constants of integration.

\section{Eigenvalue Relation}

The solutions which are given by (3.3) and (4.13)-(4.21) of the basic equations (2.4)-(2.12) must satisfy certain boundary conditions. Under the present circumstances, these boundary conditions are the following.

(i) The normal component of the velocity vector $\underline{u}$ of the fluid must be compatible with the velocity of the perturbed fluid across the fluid-tenuous interface at $r=R_{o}$. This condition gives

$$
A=\frac{-\left(\sigma+i k U_{o}\right)^{2}+\mu H_{o}^{2} m^{2} / \rho R_{o}^{2}}{k I_{m}^{\prime}(x)}
$$

where $x\left(=k R_{o}\right)$ is the nondimension longitudinal wave number.

(ii) The normal component of the magnetic field must be continuous across the fluidtenuous interface at $r=R_{o}$. This condition yields

$$
B=\frac{i \alpha H_{o}}{K_{m}^{\prime}(x)}
$$

(iii) The total magnetohydrodynamic pressure $\left(\rho \prod_{1}\right)$ interior the fluid must be discontinuous with magnetic pressure due to the electromagnetic force in the tenuous medium surrounding the fluid jet across the interface (4.10) at $r=R_{o}$ by the contribution of the capillary force. This condition reads

$$
P_{1}+R_{1} \frac{\partial P_{o}}{\partial r}+\mu\left(\underline{H}_{o} \cdot \underline{H}_{1}\right)+R_{1} \frac{\partial}{\partial r}\left(\frac{\mu}{2}\left(\underline{H}_{o} \cdot \underline{H}_{o}\right)\right)-\mu\left(\underline{H}_{o} \cdot \underline{H}_{1}\right)^{t n}-R_{1} \frac{\partial}{\partial r}\left(\frac{\mu}{2}\left(\underline{H}_{o} \cdot \underline{H}_{o}\right)^{t n}\right)=P_{1 s} .
$$

Upon substitution from (3.3)-(5.2) into (5.3), following eigenvalue relation is derived

$$
\left(\sigma+i k U_{o}\right)^{2}=\frac{\mu H_{o}^{2}}{\rho R_{o}^{2}}\left[-m^{2}+\alpha^{2} x^{2} \frac{K_{m}(x) I_{m}^{\prime}(x)}{K_{m}^{\prime}(x) I_{m}(x)}\right]+\left(\frac{T}{\rho R_{o}^{3}}\right)\left(\frac{x I_{m}^{\prime}(x)}{I_{m}(x)}\right)\left(1-m^{2}-x^{2}\right) .
$$

\section{Discussions}

Equation (5.4) is the desired capillary dispersion relation of a streaming fluid column pervaded by varying transverse magnetic field (cf. (2.1)) and surrounded by uniform magnetic field. This relation is valid for all axisymmetric and nonaxisymmetric perturbation modes. It relates the growth rate $\sigma$ with the wavenumbers $k$ and $m$, the problem parameters $\mu, H_{o}, \rho, R_{o}$ and the speed $U_{o}$ of the streaming fluid and with the modified Bessel functions $I_{m}$ and $K_{m}$ and their derivatives. 
For axisymmetric perturbation $(m=0)$, the general dispersion (5.4) reduces to

$$
\left(\sigma+i k U_{o}\right)^{2}=\left(\frac{-\mu H_{o}^{2}}{\rho R_{o}^{2}}\right)\left(\alpha^{2} x^{2}\right) \frac{K_{0}(x) I_{1}(x)}{K_{1}(x) I_{0}(x)}+\left(\frac{T}{\rho R_{o}^{3}}\right)\left(\frac{x I_{1}(x)}{I_{0}(x)}\right)\left(1-x^{2}\right) .
$$

For the lowest non-symmetric perturbation mode $(m=1)$, the dispersion relation (5.4) yields

$$
\left(\sigma+i k U_{o}\right)^{2}=\frac{\mu H_{o}^{2}}{\rho R_{o}^{2}}\left[-1+\alpha^{2} x^{2} \frac{K_{1}(x) I_{1}^{\prime}(x)}{K_{1}^{\prime}(x) I_{1}(x)}\right]-\left(\frac{T}{\rho R_{o}^{3}}\right)\left(\frac{x^{3} I_{1}^{\prime}(x)}{I_{1}(x)}\right) .
$$

For the higher nonaxisymmetric perturbation $m \geq 2$, the eigenvalue relation of such case may be easily obtained from (5.4) as $m \geq 2$.

By the use of the recurrence relations (cf. [12])

$$
\begin{gathered}
2 I_{m}^{\prime}(x)=-I_{m+1}(x)+I_{m-1}(x), \\
2 K_{m}^{\prime}(x)=-K_{m-1}(x)-K_{m+1}(x),
\end{gathered}
$$

and that $I_{m}(x)$ is monotonically increasing and positive definite for every nonzero value of $x$, we have

$$
I_{m}(x)>0 .
$$

The function $K_{m}(x)$ is monotonically decreasing but never negative for since $x \neq 0$ values

$$
K_{m}(x)>0 .
$$

Upon using (6.4) and (6.5) for the relations (6.3), we get

$$
I_{m}^{\prime}(x)>0, \quad K_{m}^{\prime}(x)<0,
$$

for all axisymmetric and nonaxisymmetric perturbation modes $m \geq 0$. By an appeal to the recurrence relations (6.3) and the inequalities (6.6) for the stability criterion (5.4), we deduce the following.

(1) The toroidal varying magnetic field interior the fluid has no influence at all on the stability of the fluid column.

(2) The uniform exterior magnetic field is stabilizing for all short and long wavelengths in all kinds of perturbation $m \geq 0$.

(3) The streaming is strongly destabilizing.

Therefore, the magnetohydrodynamic streaming fluid column is not completely stable in the axisymmetric mode $m=0$, but there will be exist some unstable domains. 
The stability discussions for the case of the lowest nonaxisymmetric mode $(m=1)$, may be carried out by utilizing the relation (6.2). It is found that both the interior toroidal and exterior axial magnetic fields are stabilizing. Note that the streaming has a strong destabilizing effect.

Therefore, the present model is (MHD) unstable in the lowest nonaxisymmetric mode $(m=1)$ of perturbation.

In a similar way, one may show that the fluid column is MHD unstable for any higher nonaxisymmetric mode $m \geq 2$ of perturbation.

Physically, the stabilizing effect of the exterior magnetic field is expected because it has been assumed that the pervading magnetic field is uniform.

Moreover, the stabilizing effect of the toroidal magnetic field in the fluid region is due to the influence of Lorentz force that comes out from the interaction of the magnetic induction and the electric current produced due to the pervading magnetic field. Indeed such electromagnetic force causes the following stresses [7].

The magnetic pressure $(\mu / 2)(\underline{H} \cdot \underline{H})$ per unit area acting in all directions of the fluid (resistivity is neglected here) and an equal magnetic tension $(\mu / 2)(\underline{H} \cdot \underline{H})$ per unit area acting along the magnetic lines of force. Due to these stresses the lines of force are able to endow the fluid with a sort of rigidity. The magnetic fields exert strong influence not only to the axisymmetric mode $(m=0)$ that causes only the bending of the magnetic lines of force but also to nonaxisymmetric modes that lead to twisting of the lines of force.

\section{Numerical Analysis}

The numerical analysis has been carried out in order to identify and examine the magnetic field influence and surface tension and also the effect of the streaming on stability of the model. In addition to that the oscillation states and the transition points from these states to those of instability may be also determined for given values of the magnetic field intensity.

This has been elaborated by computing the nondimension dispersion relation

$$
\frac{\sigma}{\sqrt{T / \sigma R_{o}^{3}}}=\sqrt{\left(\frac{H_{o}}{H_{s}}\right)\left(m^{2}+\alpha^{2} x^{2} \frac{K_{m}(x) I_{m}^{\prime}(x)}{K_{m}^{\prime}(x) I_{m}(x)}\right)+\left(\frac{x I_{m}^{\prime}(x)}{I_{m}(x)}\right)\left(1-m^{2}-x^{2}\right)+U^{*}}
$$

in the computer simulation for the most dangerous sausage mode $m=0$ for the different values of $(\alpha)$ and $\left(H_{o} / H_{S}\right)$, where $H_{S}\left(=\left(T / \mu R_{o}\right)\right)$ has the dimension of magnetic field while $\sigma(=i \omega)$ is the growth rate and $\omega$ is the oscillation frequency.

The numerical data associated with $\omega /\left(T / \rho R_{o}^{3}\right)^{1 / 2}$ corresponding to the stable states and those associated with $\sigma /\left(T / \rho R_{o}^{3}\right)^{1 / 2}$ corresponding to the unstable states are collected, tabulated, and presented graphically. See Figures 2, 3, 4, and 5 . There are many features of interest in these numerical illustration. In the absence of the streaming effect it is found that the domains of instability are decreasing with increasing $\left(H_{o} / H_{S}\right)$ values even for different values of $\alpha$.

For $\alpha=1$ see Figure 2: corresponding to $h=\left(H_{o} / H_{s}\right)=0.1,0.3,0.7,1.0,1.5$, and 3.5, it is found that the unstable domains are $0 \leq x<0.9965,0 \leq x<0.9693,0 \leq x<0.8496,0 \leq x<$ $0.7307,0 \leq x<0.5436,0 \leq x<0.2083$, while the neighboring stable domains, respectively, are $0.9965 \leq x<\infty, 0.9693 \leq x<\infty, 0.8496 \leq x<\infty, 0.7307 \leq x<\infty, 0.5436 \leq x<\infty$, and $0.2083 \leq x<\infty$. 


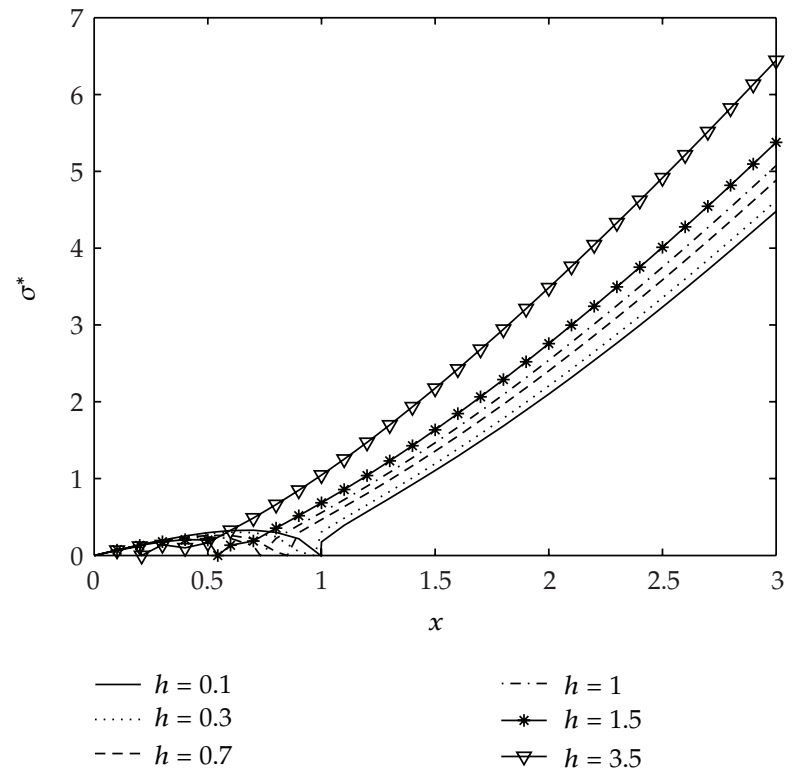

Figure 2: MHD stable and unstable domains as $\alpha=1, m=0, U^{*}=0$.

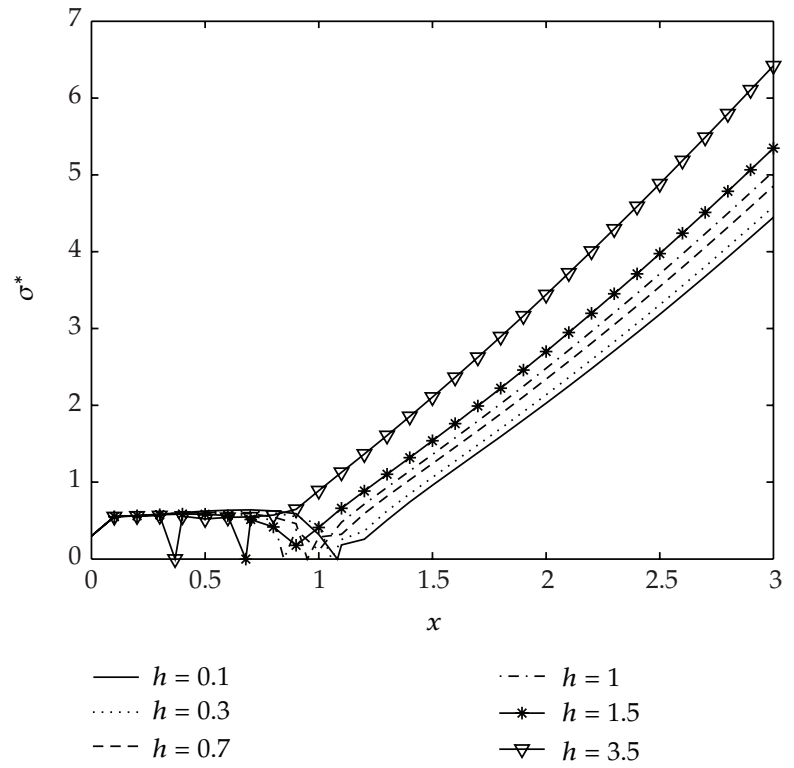

Figure 3: MHD stable and unstable domains as $\alpha=1, m=0, U^{*}=0.3$.

For $\left(\alpha, U^{*}\right)=(1,0.3)$ : corresponding to $h=\left(H_{o} / H_{s}\right)=0.1,0.3,0.7,1.0,1.5$, and 3.5, it is found that the unstable domains are $0 \leq x<1.0819,0 \leq x<1.0561,0 \leq x<0.9491,0 \leq$ $x<0.8464,0 \leq x<0.6791,0 \leq x<0.3678$, while the neighboring stable domains, respectively, are $1.0819 \leq x<\infty, 1.0561 \leq x<\infty, 0.9491 \leq x<\infty, 0.8464 \leq x<\infty, 0.6791 \leq$ $x<\infty, 0.3678 \leq x<\infty$, see Figure 3. 


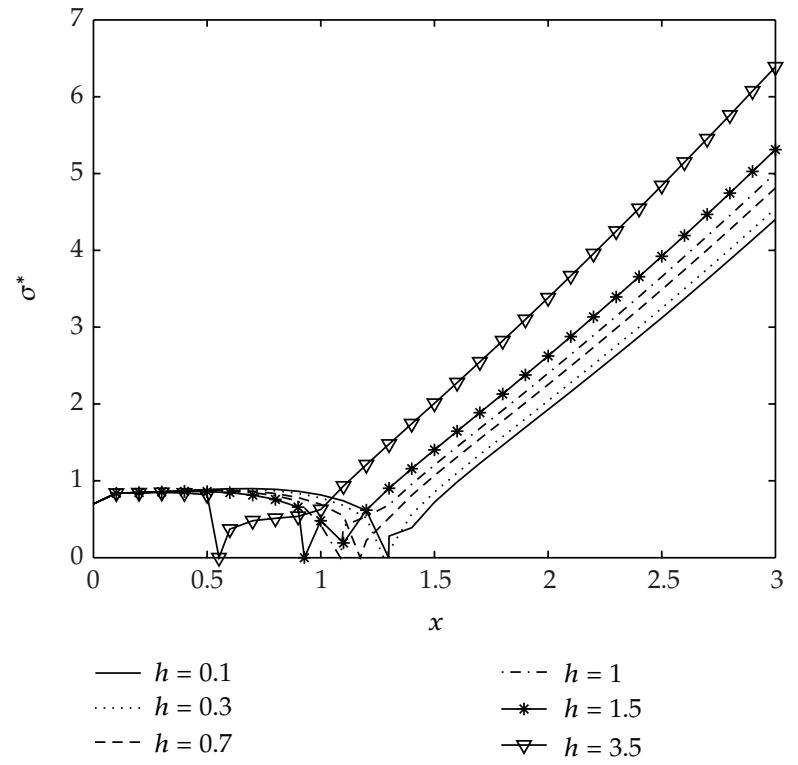

Figure 4: MHD stable and unstable domains as $\alpha=1, m=0, U^{*}=0.7$.

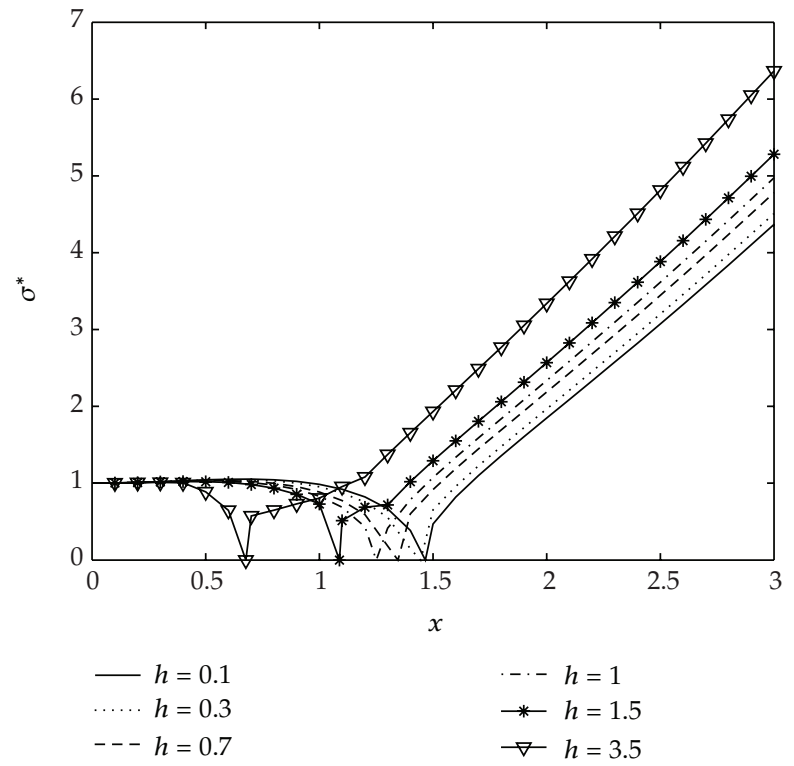

Figure 5: MHD stable and unstable domains as $\alpha=1, m=0, U^{*}=1.0$.

For $\left(\alpha, U^{*}\right)=(1,0.7)$ : corresponding to $h=\left(H_{o} / H_{s}\right)=0.1,0.3,0.7,1.0,1.5$, and 3.5, it is found that the unstable domains are $0 \leq x<1.2985,0 \leq x<1.2769,0 \leq x<1.1735,0 \leq$ $x<1.0867,0 \leq x<0.9277,0 \leq x<0.5519$, while the neighboring stable domains, respectively, are $1.2985 \leq x<\infty, 1.2769 \leq x<\infty, 1.1735 \leq x<\infty, 1.0867 \leq x<\infty, 0.9277 \leq$ $x<\infty, 0.5519 \leq x<\infty$, see Figure 4. 
For $\left(\alpha, U^{*}\right)=(1,1.0)$ : corresponding to $h=\left(H_{o} / H_{S}\right)=0.1,0.3,0.7,1.0,1.5$, and 3.5, it is found that the unstable domains are $0 \leq x<1.4661,0 \leq x<1.4453,0 \leq x<1.3455,0 \leq$ $x<1.2500,0 \leq x<1.0882,0 \leq x<0.6765$, while the neighboring stable domains, respectively, are $1.4661 \leq x<\infty, 1.4453 \leq x<\infty, 1.3455 \leq x<\infty, 1.2500 \leq x<\infty, 1.0882 \leq$ $x<\infty, 0.6765 \leq x<\infty$, see Figure 5 .

\section{Conclusion}

From the presented numerical results, we may deduce the following. For the same value of $U^{*}$ it is found that the unstable domains are decreasing with increasing of $h$ values. This means that the influence of magnetic field has a stabilizing effect for all short and long wavelengths. For the same value of $h$, it is found that the unstable domains are increasing with increasing of $U^{*}$ values. This means that the streaming is strongly destabilizing.

\section{References}

[1] B. J. W. S. Rayleigh, The Theory of Sound, Dover, New York, NY, USA, 2nd edition, 1945.

[2] S. Chandrasekhar, Hydrodynamic and Hydromagnetic Stability, Dover, New York, NY, USA, 1981.

[3] P. H. Robert, An Introduction to MHD, Longman, London, UK, 1967.

[4] L. Chenng, "Instability of a gas jet in liquid," Physics of Fluids, vol. 28, article 2614, 3 pages, 1985.

[5] J. M. Kendall, "Experiments on annular liquid jet instability and on the formation of liquid shells," Physics of Fluids, vol. 29, no. 7, pp. 2086-2094, 1986.

[6] P. G. Drazin and W. H. Reid, Hydrodynamic Stability, Cambridge Mathematical Library, Cambridge University Press, Cambridge, Mass, USA, 2nd edition, 2004.

[7] A. E. Radwan, "Effect of magnetic fields on the capillary instability of an annular liquid jet," Journal of Magnetism and Magnetic Materials, vol. 72, no. 2, pp. 219-232, 1988.

[8] A. E. Radwan, M. A. Elogail, and N. E. Elazab, "Large hydromagnetic axisymmetric instability of a streaming gas cylinder surrounded by bounded fluid with non uniform field," Kyungpook Mathematical Journal, vol. 47, no. 4, pp. 455-471, 2007.

[9] A. E. Radwan and A. A. Hasan, "Axisymmetric electrogravitational stability of fluid cylinder ambient with transverse varying oscillating field," IAENG International Journal of Applied Mathematics, vol. 38, no. 3, pp. 113-120, 2008.

[10] A. E. Radwan and A. A. Hasan, "Magneto hydrodynamic stability of self-gravitational fluid cylinder," Applied Mathematical Modelling, vol. 33, no. 4, pp. 2121-2131, 2009.

[11] A. A. Hasan, "Electrogravitational stability of oscillating streaming fluid cylinder," Physica B, vol. 406, no. 2, pp. 234-240, 2011.

[12] M. Abramowitz and I. Stegun, Handbook of Mathematical Functions, Dover, New York, NY, USA, 1970. 


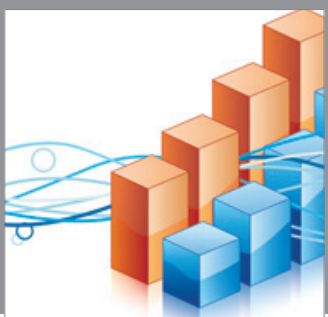

Advances in

Operations Research

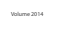

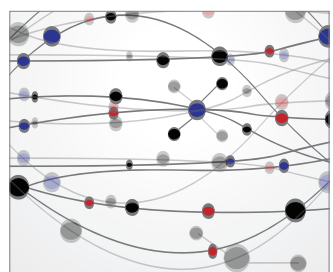

\section{The Scientific} World Journal
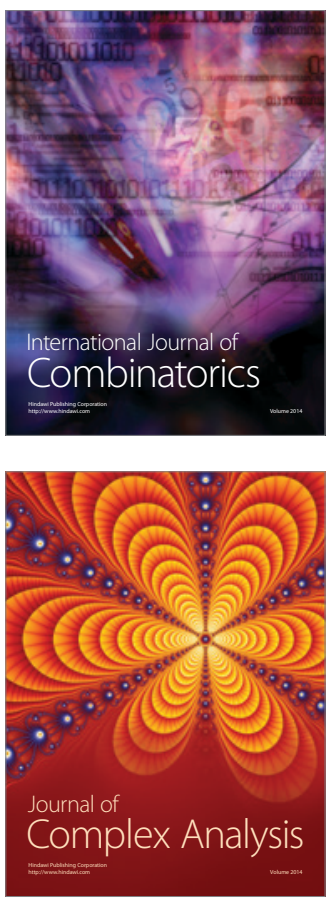

International Journal of

Mathematics and

Mathematical

Sciences
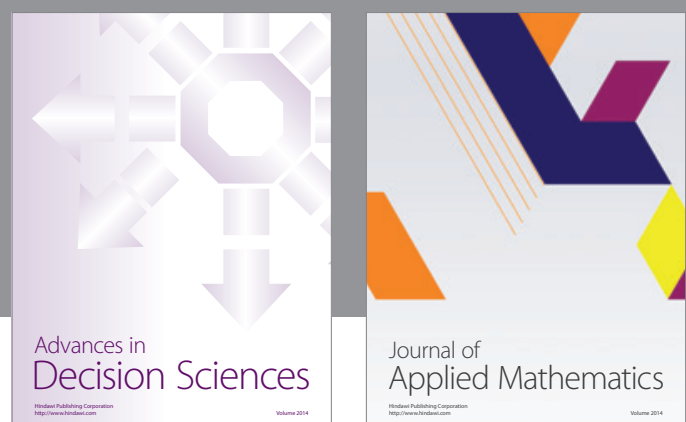

Journal of

Applied Mathematics
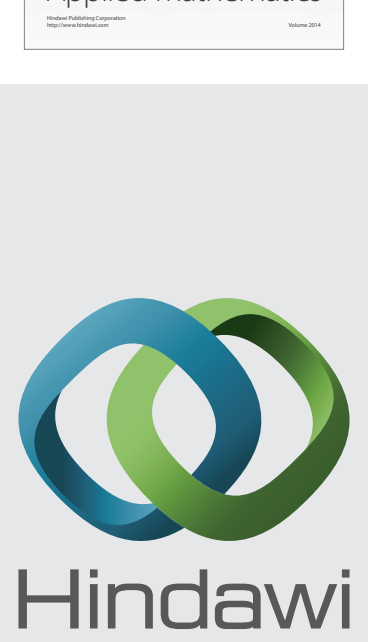

Submit your manuscripts at http://www.hindawi.com
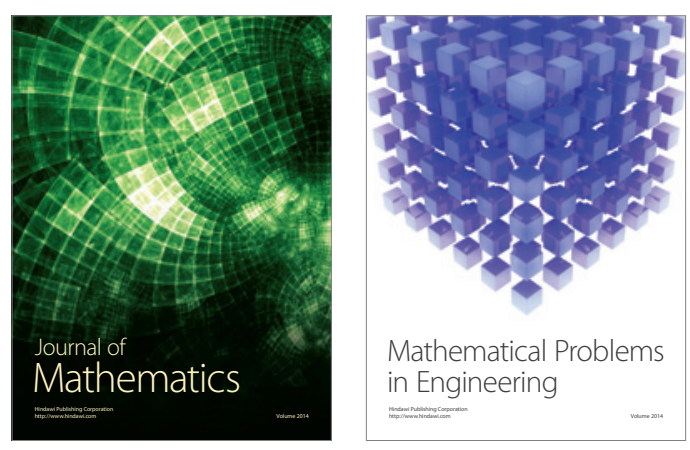

Mathematical Problems in Engineering
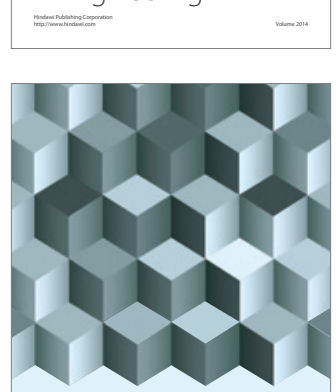

Journal of

Function Spaces
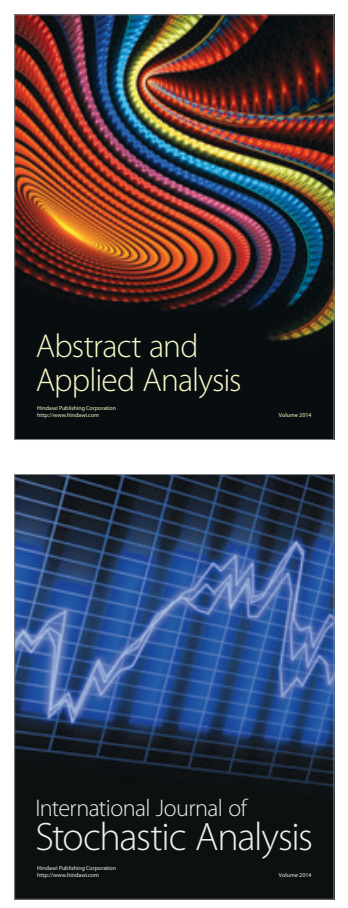

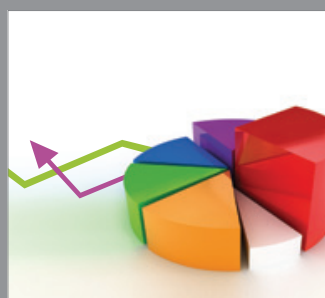

ournal of

Probability and Statistics

Promensencen
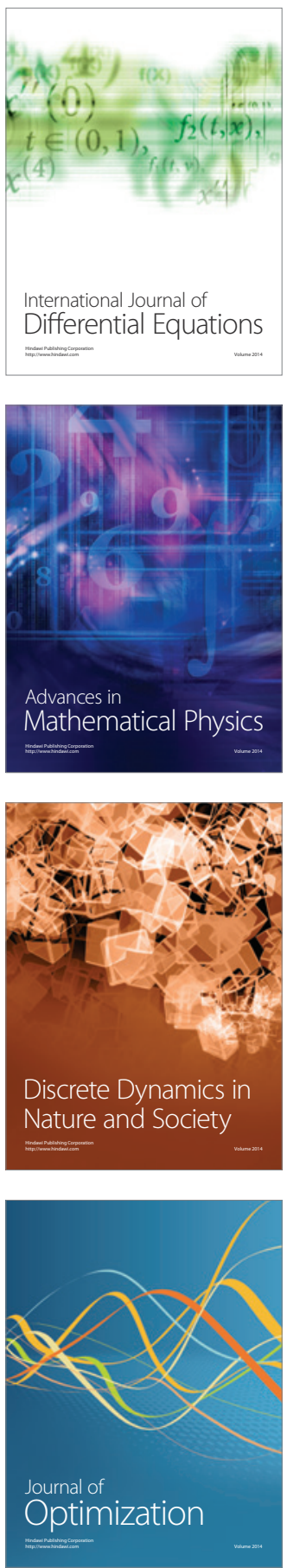